TAIWANESE JOURNAL OF MATHEMATICS

Vol. 13, No. 3, pp. 855-870, June 2009

This paper is available online at http://www.tjm.nsysu.edu.tw/

\title{
ASYMPTOTICS OF THE LANDAU CONSTANTS AND THEIR RELATIONSHIP WITH HYPERGEOMETRIC FUNCTIONS
}

\author{
Djurdje Cvijovic and H. M. Srivastava*
}

Abstract. We examine the Landau constants defined by

$$
G_{n}:=\sum_{m=0}^{n} \frac{1}{2^{4 m}}\left(\begin{array}{c}
2 m \\
m
\end{array}\right)^{2} \quad(n=0,1,2, \cdots)
$$

by making use of the celebrated Ramanujan formula expressing $G_{n}$ in terms of the Clausenian ${ }_{3} F_{2}$ hypergeometric series. It is shown that it could be used to deduce other, mostly new, Ramanujan type formulas for the Landau constants involving the terminating and non-terminating hypergeometric series. In addition, by this approach we derive once again, in a simple and unified manner, almost all of the known results and also establish several new results for $G_{n}$. These new results include (for example) the generating function and asymptotic expansions and estimates for $G_{n}$.

\section{LANDAu Constants}

The normalized central binomial coefficients:

$$
\mu_{m} \quad\left(m \in \mathbb{N}_{0}:=\mathbb{N} \cup\{0\} ; \mathbb{N}:=\{1,2,3, \cdots\}\right)
$$

can be variously defined in terms of the binomial coefficients and factorials as follows:

$$
\mu_{m}=\frac{1}{2^{2 m}}\left(\begin{array}{c}
2 m \\
m
\end{array}\right)=(-1)^{m}\left(\begin{array}{c}
-\frac{1}{2} \\
m
\end{array}\right)=\frac{(2 m) !}{\left(2^{m} \cdot m !\right)^{2}} \quad\left(m \in \mathbb{N}_{0}\right)
$$

Received and accepted March 26, 2008.

Communicated by J. C. Yao.

2000 Mathematics Subject Classification: Primary 11Y60, 26D15, 41A60; Secondary 30B10, 33C05. Key words and phrases: Landau constants, Inequalities, Psi function, Ramanujan formula, Generalized Gauss hypergeometric functions, Generating functions, Asymptotic expansions and estimates, Clausenian hypergeometric function, Central binomial coefficients and central factorials, Bernoulli polynomials.

*Corresponding author. 
or, equivalently, as follows:

$$
\mu_{0}=1 \quad \text { and } \quad \mu_{m}=\frac{(2 m-1) ! !}{(2 m) ! !} \quad(m \in \mathbb{N})
$$

in terms of double factorials, which are given by

$0 ! !:=1, \quad(2 n) ! !=2 \cdot 4 \cdot 6 \cdots(2 n), \quad$ and $\quad(2 n-1) ! !=1 \cdot 3 \cdot 5 \cdots(2 n-1) \quad(n \in \mathbb{N})$

or, alternatively, as follows:

$$
\mu_{m}=\frac{1}{\sqrt{\pi}} \frac{\Gamma\left(m+\frac{1}{2}\right)}{\Gamma(m+1)}=\frac{\Gamma\left(m+\frac{1}{2}\right)}{\Gamma\left(\frac{1}{2}\right) \Gamma(m+1)}=\frac{\left(\frac{1}{2}\right)_{m}}{(1)_{m}} \quad\left(m \in \mathbb{N}_{0}\right)
$$

in terms of the familiar Gamma function $\Gamma(z)$ and the Pochhammer symbol $(\lambda)_{n}$ defined (for $\lambda \in \mathbb{C}$ ) by [17, p. 2]

$$
(\lambda)_{n}=\frac{\Gamma(\lambda+n)}{\Gamma(\lambda)}= \begin{cases}1 & (n=0) \\ \lambda(\lambda+1) \cdots(\lambda+n-1) & (n \in \mathbb{N}) .\end{cases}
$$

The numbers $G_{0}, G_{1}, G_{2}, \cdots$ given by (see, for instance, [1, p. 216], [4, p. 159], [8, p. 252], [20, p. 310] and [21])

$$
G_{n}=\sum_{m=0}^{n} \mu_{m}^{2}=1+\left(\frac{1}{2}\right)^{2}+\left(\frac{1 \cdot 3}{2 \cdot 4}\right)^{2}+\cdots+\left(\frac{1 \cdot 3 \cdot 5 \cdots(2 n-1)}{2 \cdot 4 \cdot 6 \cdots(2 n)}\right)^{2}
$$

are known as the Landau constants (see also a recent investigation by Alzer et al. [2] involving various other important mathematical constants). The values of the first few of these constants are given by

$$
\begin{array}{rlrl}
G_{0} & =1, \quad G_{1}=\frac{5}{4}=1.25, & G_{2}=\frac{89}{64}=1.3906250 \\
G_{3}=\frac{381}{256}=1.488281250, & G_{4}=\frac{25609}{16384}=1.563049316406250 \\
G_{5}=\frac{106405}{65536}=1.62361145019531250, &
\end{array}
$$

and

$$
G_{6}=\frac{1755841}{1048576}=1.674500465393066406250 .
$$

Now we choose to recall here the following two classical results concerning the Landau constants. 
Theorem A. (Landau, 1913, [1, p. 215] and [10]). Let

$$
f(z)=\sum_{m=0}^{\infty} a_{m} z^{m}
$$

be a complex function of complex argument which is analytic inside the unit disk, continuous on its boundary and satisfies the following inequality:

$$
|f(z)|<1 \quad \text { whenever } \quad|z|<1 .
$$

Then

$$
\left|\sum_{m=0}^{n} a_{m}\right| \leqq G_{n},
$$

$G_{n}$ being the constants defined as in (5).

Moreover, if $T_{n}(f)$ is a polynomial operator associated with $f(z)$, then its norm is given by

$$
\left\|T_{n}\right\|=G_{n} .
$$

Theorem B. (Ramanujan, 1913, [15, p. 351] and [19, p. 82]). Let ${ }_{3} F_{2}$ denote the generalized hypergeometric function defined as in Section 2 below. If $n \in \mathbb{N}_{0}$, then

$$
\begin{aligned}
& \left(\frac{\Gamma(n+1)}{\Gamma\left(n+\frac{3}{2}\right)}\right)^{2} \cdot G_{n}=\frac{1}{n+1}{ }_{3} F_{2}\left[\begin{array}{r}
\frac{1}{2}, \frac{1}{2}, n+1 ; \\
1, n+2 ;
\end{array}\right] \\
& =\frac{1}{n+1}+\left(\frac{1}{2}\right)^{2} \frac{1}{n+2}+\left(\frac{1 \cdot 3}{2 \cdot 4}\right)^{2} \frac{1}{n+3}+\left(\frac{1 \cdot 3 \cdot 5}{2 \cdot 4 \cdot 6}\right)^{2} \frac{1}{n+4}+\cdots .
\end{aligned}
$$

In his first letter to Hardy, dated 16 January 1913, Ramanujan stated the formula (6) without proof. When letters of Ramanujan to Hardy were reproduced in full with comments in Ramanujan's collected papers published in 1927 and this result became publicly known, the formula (6) as well as its various generalizations attracted a great deal of attention.

It is interesting to remark here that the Ramanujan formula (6) is rarely cited in the newer literature on the Landau constants $G_{n}$. Here, we make use of this formula and exploit to the full its connection with the theory of the generalized hypergeometric functions and deduce other, mostly new, Ramanujan type formulas fortheLandauconstants involving theterminating and non-terminating hypergeometric series. Moreover, by this approach we again derive, in a simple and unified manner, almost all of the known results for $G_{n}$ and also establish various new results for $G_{n}$ such as (for example) the generating function (see, for details, [18]) as well as asymptotic expansions and estimates for the Landau constants $G_{n}$. 


\section{Landau Constants and Hypergeometric Functions}

The generalized (Gauss) hypergeometric ${ }_{p} F_{q}$ is, as usual, defined by the following hypergeometric series $[17$, p. 52]:

$$
{ }_{p} F_{q}\left[\begin{array}{c}
\alpha_{1}, \alpha_{2}, \cdots, \alpha_{p} ; \\
\beta_{1}, \beta_{2}, \cdots, \beta_{q} ;
\end{array}\right]=\sum_{m=0}^{\infty} \frac{\left(\alpha_{1}\right)_{m}\left(\alpha_{2}\right)_{m} \cdots\left(\alpha_{p}\right)_{m}}{\left(\beta_{1}\right)_{m}\left(\beta_{2}\right)_{m} \cdots\left(\beta_{q}\right)_{m}} \frac{z^{m}}{m !}
$$

whenever the series converges and (by analytic continuation) elsewhere. Here $(\cdot)_{m}$ denotes the Pochhammer symbol defined by (4), $p$ and $q$ are nonnegative integers, the complex numbers $\alpha_{1}, \alpha_{2}, \cdots, \alpha_{p}$ and $\beta_{1}, \beta_{2}, \cdots, \beta_{q}$ are called, respectively, the numerator and denominator parameters, and $z$ is called the variable. The denominator parameters are not allowed to be zero or negative integers and the ${ }_{p} F_{q}$ function is symmetric in its numerator parameters, and likewise in its denominator parameters. We are concerned here only with the cases when $p=q+1$. In these instances, the series defining ${ }_{p} F_{q}$ converges when $|z|<1$ for all choices of the parameters involved. If $z=1$, the series converges for

$$
\Re\left(\beta_{1}+\beta_{2}+\cdots+\beta_{q}-\alpha_{1}-\alpha_{2}-\cdots-\alpha_{p}\right)>0 .
$$

We begin by noting that (presumably) it has not been noticed so far that the Landau constants $G_{n}$ admit a generating function. For this purpose, we need to recall the following definition $[17$, p. 49 , Eq. (33)]:

$$
K(k)=\int_{0}^{\pi / 2} \frac{d \phi}{\sqrt{1-k^{2} \sin ^{2} \phi}} \quad\left(k^{2}<1\right)
$$

of the complete elliptic integral $K(k)$ of the first kind with the (elliptic) modulus $k$.

Proposition 1. Consider the Landau constants defined by (5) and let $K(k)$ be the above-defined complete elliptic integral. Then

$$
g(x):=\frac{2}{\pi} \frac{K(x)}{1-x}=\sum_{n=0}^{\infty} G_{n} x^{n} .
$$

Demonstration. Indeed, it is not difficult to show that $g(x)$ is the generating function for $G_{n}$ by making use of the Leibniz product rule and the following well-known expansion of $K(k)$ in the Gauss hypergeometric series [17, p. 49, Eq. (33)]:

$$
\frac{2}{\pi} K(k)={ }_{2} F_{1}\left(\frac{1}{2}, \frac{1}{2} ; 1 ; k^{2}\right)=1+\sum_{m=1}^{\infty}\left(\frac{(2 m-1) ! !}{(2 m) ! !}\right)^{2} k^{2 m} \quad\left(k^{2}<1\right) .
$$


We shall next record another interesting connection between $G_{n}$ and $K(k)(c f$. Watson [20, p. 314, Sect. 3] and Dutka [5, p. 472, Eq. (2.4)]).

Proposition 2. If $n \in \mathbb{N}_{0}$, then

$$
G_{n}=\frac{4}{\pi}\left(\frac{\Gamma\left(n+\frac{3}{2}\right)}{\Gamma(n+1)}\right)^{2} \int_{0}^{1} k^{2 n+1} K(k) d k .
$$

Demonstration. Clearly, in view of the above series expansion of $K(k)$, the Ramanujan result (6) could be recast as follows:

$$
\begin{aligned}
& G_{n} \cdot\left(\frac{\Gamma(n+1)}{\Gamma\left(n+\frac{3}{2}\right)}\right)^{2} \\
= & \frac{1}{n+1}+\left(\frac{1}{2}\right)^{2} \frac{1}{n+2}+\left(\frac{1 \cdot 3}{2 \cdot 4}\right)^{2} \frac{1}{n+3}+\left(\frac{1 \cdot 3 \cdot 5}{2 \cdot 4 \cdot 6}\right)^{2} \frac{1}{n+4}+\cdots \\
= & 2 \int_{0}^{1}\left[1+\left(\frac{1}{2}\right)^{2} k^{2}+\left(\frac{1 \cdot 3}{2 \cdot 4}\right)^{2} k^{4}+\left(\frac{1 \cdot 3 \cdot 5}{2 \cdot 4 \cdot 6}\right)^{2} k^{6}+\cdots\right] k^{2 n+1} d k \\
= & \frac{4}{\pi} \int_{0}^{1} k^{2 n+1} K(k) d k,
\end{aligned}
$$

showing that the assertion (9) holds true.

We shall now establish four Ramanujan type formulas for the Landau constants of which only one can be found in the literature. Proposition 6 was recently proved by Eisinberg et al. [6, p. 59, Eq. (7)] by using some rather involved argument and without appealing to the theory of generalized hypergeometric functions.

Proposition 3. The following explicit hypergeometric representation holds true:

$$
G_{n}=\frac{4}{\pi}\left(\frac{\Gamma\left(n+\frac{3}{2}\right)}{\Gamma(n+1)}\right)^{2}{ }_{3} F_{2}\left[\begin{array}{r}
-n, 1,1 ; \\
\frac{3}{2}, \frac{3}{2} ; 1
\end{array}\right] \quad\left(n \in \mathbb{N}_{0}\right) .
$$

Demonstration. The above-proposed identity follows at once from (6) upon making use of the following known transformation [14, p. 533, Entry 7.4.4.2]:

$$
\begin{gathered}
{ }_{3} F_{2}\left[\begin{array}{r}
a, b, c ; \\
d, e ;
\end{array}\right]=\frac{\Gamma(d) \Gamma(e) \Gamma(s)}{\Gamma(a) \Gamma(b+s) \Gamma(c+s)}{ }_{3} F_{2}\left[\begin{array}{c}
d-a, e-a, s ; \\
b+s, c+s ;
\end{array}\right] \\
(s:=d+e-a-b-c ; \min \{\Re(a), \Re(s)\}>0) .
\end{gathered}
$$


Proposition 4. If $n \in \mathbb{N}_{0}$, then

$$
G_{n}=\frac{(2 n+1) ! !}{(2 n) ! !}{ }_{3} F_{2}\left[\begin{array}{c}
-n, \frac{1}{2}, \frac{1}{2} ; \\
\frac{3}{2}, 1 ;
\end{array}\right]
$$

Demonstration. In order to verify this formula, it suffices to use Proposition 3 and the following transformation [14, p. 533, Entry 7.4.4.1]:

$$
\begin{gathered}
{ }_{3} F_{2}\left[\begin{array}{c}
a, b, c ; \\
1 \\
d, e ;
\end{array}\right]=\frac{\Gamma(d) \Gamma(d+e-a-b-c)}{\Gamma(d+e-a-b) \Gamma(d-c)}{ }_{3} F_{2}\left[\begin{array}{c}
e-a, e-b, c ; \\
d+e-a-b, e ;
\end{array}\right] \\
(\min \{\Re(d+e-a-b-c), \Re(d-c)\}>0) .
\end{gathered}
$$

Further, in a similar fashion, we obtain the next two results. Indeed, upon combining (12) and (11), we arrive at Proposition 5, while Proposition 6 is obtained by making use of (12) and the following transformation $[14$, p. 546, Entry 7.4.5.2]:

$$
\begin{aligned}
& { }_{3} F_{2}\left[\begin{array}{rr}
a, b, c ; \\
1+a-b, 1+a-c ;
\end{array}\right] \\
= & \frac{\Gamma(1+a-b) \Gamma(1+a-c)}{\Gamma(1+a) \Gamma(1+a-b-c)}{ }_{3} F_{2}\left[\begin{array}{cc}
\frac{1}{2}, b, c ; \\
\frac{a}{2}+1, \frac{a+1}{2} ;
\end{array}\right] .
\end{aligned}
$$

Proposition 5. If $n \in \mathbb{N}_{0}$, then

$$
G_{n}=\frac{1}{2(n+1)}\left(\frac{(2 n+1) ! !}{(2 n) ! !}\right)^{2}{ }_{3} F_{2}\left[\begin{array}{c}
\frac{1}{2}, 1, n+\frac{3}{2} ; \\
\frac{3}{2}, n+2 ;
\end{array}\right]
$$

Proposition 6. If $n \in \mathbb{N}_{0}$, then

$$
G_{n}=\frac{4}{\pi(n+1)}\left(\frac{\Gamma\left(n+\frac{3}{2}\right)}{\Gamma(n+1)}\right)^{2}{ }_{3} F_{2}\left[\begin{array}{cc}
-n, 1, \frac{1}{2} ; & -1 \\
\frac{3}{2}, 2+n ;
\end{array}\right]
$$

Remark. It is well-known that, in general, the ${ }_{p} F_{q}$ series terminates and, therefore, is a polynomial if a numerator parameter is a negative integer or zero, provided that no denominator parameter is a negative integer or zero. 
The series in the original Ramanujan formula (6) is nonterminating, while the ${ }_{3} F_{2}$ series given by (10), (12) and (14) are terminating, and they can be rewritten by making use of the following finite sum:

$$
{ }_{3} F_{2}\left[\begin{array}{r}
-n, \alpha, \beta ; \\
\gamma, \delta ;
\end{array}\right]=\sum_{m=0}^{n} \frac{(-n)_{m}(\alpha)_{m}(\beta)_{m}}{(\gamma)_{m}(\delta)_{m}(1)_{m}} x^{m} .
$$

Thus, in light of this and the following identities (see, for instance, [14, p. 758]):

$$
\begin{aligned}
& (-n)_{m}=(-1)^{m} m !\left(\begin{array}{c}
n \\
m
\end{array}\right), \quad(n)_{m}=\frac{(n+m-1) !}{(n-1) !} \\
& \left(\frac{1}{2}\right)_{m}=\frac{1}{2^{2 m}} \frac{(2 m) !}{m !}, \quad \text { and } \quad\left(\frac{3}{2}\right)_{m}=\frac{1}{2^{2 m}} \frac{(2 m+1) !}{m !},
\end{aligned}
$$

starting from (10), (12) and (14), after some simple algebra, we can write down a number of formulas for $G_{n}$. We list a few examples in Proposition 7: Part (a), Parts (b) and (c), and Parts (d), (e) and (f) follow from (10), (12), and (14), respectively.

Proposition 7. If $n \in \mathbb{N}_{0}$, then

$$
\begin{aligned}
& \text { (a) } \quad G_{n}=\left(\frac{(2 n+1) ! !}{(2 n) ! !}\right)^{2} \sum_{m=0}^{n} \frac{(-1)^{m} 2^{4 m}}{(2 m+1)^{2}}\left(\begin{array}{c}
n \\
m
\end{array}\right)\left(\begin{array}{c}
2 m \\
m
\end{array}\right)^{-2} ; \\
& \text { (b) } \quad G_{n}=\frac{2 n+1}{2^{2 n}}\left(\begin{array}{c}
2 n \\
n
\end{array}\right) \sum_{m=0}^{n} \frac{(-1)^{m}}{(2 m+1) 2^{2 m}}\left(\begin{array}{c}
n \\
m
\end{array}\right)\left(\begin{array}{c}
2 m \\
m
\end{array}\right) ; \\
& \text { (c) } \quad G_{n}=\frac{2 n+1}{2^{2 n}}\left(\begin{array}{c}
2 n \\
n
\end{array}\right) \sum_{m=0}^{n} \frac{1}{(2 m+1)}\left(\begin{array}{c}
n \\
m
\end{array}\right)\left(\begin{array}{c}
-\frac{1}{2} \\
m
\end{array}\right) ; \\
& \text { (d) } \quad G_{n}=2(-1)^{n+1} \sum_{m=0}^{n}\left(\begin{array}{c}
n+\frac{1}{2} \\
m
\end{array}\right)\left(\begin{array}{c}
n-\frac{1}{2}-m \\
2 n+1-m
\end{array}\right) ; \\
& \text { (e) } \quad G_{n}=\frac{(2 n+1)^{2}}{n+1}\left(\begin{array}{c}
-\frac{1}{2} \\
n
\end{array}\right)^{2} \sum_{m=0}^{n} \frac{1}{2 m+1}\left(\begin{array}{c}
n \\
m
\end{array}\right)\left(\begin{array}{c}
n+m+1 \\
m
\end{array}\right)^{-1} ; \\
& \text { (f) } \quad G_{n}=\frac{(2 n+1) !}{\left(2^{2 n} n !\right)^{2}} \sum_{m=0}^{n}\left(\begin{array}{c}
2 n+1 \\
m
\end{array}\right) \frac{1}{2(n-m)+1} .
\end{aligned}
$$

It should be noted that the formula (f) in Proposition 7 has been known for a long time (see Schönhage [16, p. 64] and Mills and Smith [12, pp. 115 and 116]) and that the formulas (d) and (e) were recently deduced by Eisinberg at al. [6, p. 61, Eqs. (17) and (18)], while the formulas (a), (b) and (c) are (presumably) new. 
It is known that, in a ${ }_{3} F_{2}$ form, if a numerator parameter exceeds any denominator parameter by a positive integer, say $n$, the ${ }_{3} F_{2}$ series may be expressed as the sum of $n+1$ hypergeometric ${ }_{2} F_{1}$ series (see, for instance, [14, p. 439, Entry 7.2.3.15]). If the latter can be summed, then so can the ${ }_{3} F_{2}$ be. We note that the ${ }_{3} F_{2}$ series given by (13) exhibits this property and we here present a very simple and straightforward derivation of the identity given by Proposition 8 in sharp contrast to two rather lengthy and complicated proofs given by Montaldi and Zucchelli [13, pp. 562 and 563].

Proposition 8. The following identity holds true:

$$
G_{n}=\frac{\left(\frac{3}{2}\right)_{n}}{n !} \sum_{m=0}^{n} \frac{\left(\frac{1}{2}\right)_{m}}{m !} \frac{1}{2(n-m)+1} .
$$

Demonstration. Indeed, by making use of the transformation [14, p. 439, Entry 7.2.3.15]) in conjunction with the Gauss summation theorem [14, p. 489, Entry 7.3.5.2]:

$$
{ }_{2} F_{1}\left[\begin{array}{c}
a, b ; \\
1 \\
c ;
\end{array}\right]=\frac{\Gamma(c) \Gamma(c-a-b)}{\Gamma(c-a) \Gamma(c-b)} \quad(\Re(c-a-b)>0)
$$

in Proposition 5, we have

$$
\begin{aligned}
G_{n} & =\frac{1}{2(n+1)}\left(\frac{\left(\frac{3}{2}\right)_{n}}{n !}\right)^{2} \sum_{m=0}^{n}\left(\begin{array}{c}
n \\
m
\end{array}\right) \frac{\left(\frac{1}{2}\right)_{m}(1)_{m}}{\left(\frac{3}{2}\right)_{m}(n+2)_{m}}{ }_{2} F_{1}\left[\begin{array}{c}
\frac{1}{2}+m, 1+m ; \\
n+2+m ;
\end{array}\right] \\
& =\frac{1}{2} \frac{\left(\frac{3}{2}\right)_{n}}{n !} \sum_{m=0}^{n} \frac{1}{2 m+1} \frac{\left(\frac{3}{2}\right)_{n}}{(n-m) !} \frac{\Gamma\left(n-m+\frac{1}{2}\right)}{\Gamma\left(n+\frac{3}{2}\right)} \\
& =\frac{1}{2} \frac{\left(\frac{3}{2}\right)_{n}}{n !} \sum_{m=0}^{n} \frac{1}{2(n-m)+1} \frac{\left(\frac{3}{2}\right)_{n}}{m !} \frac{\Gamma\left(m+\frac{1}{2}\right)}{\Gamma\left(n+\frac{3}{2}\right)} \\
& =\frac{\left(\frac{3}{2}\right)_{n}}{n !} \sum_{m=0}^{n} \frac{\left(\frac{1}{2}\right)_{m}}{m !} \frac{1}{2(n-m)+1} .
\end{aligned}
$$

A particularly short proof of the next proposition can be obtained if we consider $G_{n}$ as partial sums of the (divergent) hypergeometric series as follows [ $c f$. Eqs. (3) and (5)]:

$$
G_{n}=\sum_{m=0}^{n} \frac{\left(\frac{1}{2}\right)_{m}\left(\frac{1}{2}\right)_{m}}{(1)_{m}} \frac{1}{m !}
$$


and then apply the following known result [11, p. 109, Eq. (34)]:

$$
\begin{aligned}
& { }_{2} F_{1}\left[\begin{array}{c}
a, b ; \\
a+b ;
\end{array}\right]_{n}:=\sum_{m=0}^{n} \frac{(a)_{m}(b)_{m}}{(a+b)_{m}} \frac{1}{m !}=\frac{\Gamma(a+b)}{\Gamma(a) \Gamma(b)} \\
& \cdot\left(\psi(b+n+1)-\psi(a)-\psi(b)+\psi(1)-\frac{b(1-a)}{b+n+1}{ }_{4} F_{3}\left[\begin{array}{c}
b+1,2-a, 1,1 ; \\
b+n+2,2,2 ;
\end{array}\right]\right),
\end{aligned}
$$

where $\psi(x)$ is the Psi (or Digamma) function defined by [17, p. 13, Eq. (1)]

$$
\psi(z)=\frac{\Gamma^{\prime}(z)}{\Gamma(z)}=\frac{d}{d z}\{\log \Gamma(z)\} .
$$

We note that Eq. (15) below is proven by different arguments in several places (see Dutka [5, p. 474, Eq. (3.4)], Mills and Smith [12, p. 114, Theorem 2] and Cvijovic and Klinowski [4, p. 161, Theorem 1]).

Proposition 9. Let

$$
\gamma=-\psi(1)=0.577215664901532860606512 \cdots
$$

denote the Euler-Mascheroni constant and let $\psi(x)$ be the Psi function. Then

$$
G_{n}=\frac{1}{\pi} \psi\left(n+\frac{3}{2}\right)+\frac{1}{\pi}(\gamma+4 \log 2)-\delta_{n},
$$

where

$$
\delta_{n}=\frac{1}{\pi} \frac{1}{4\left(n+\frac{3}{2}\right)}{ }_{4} F_{3}\left[\begin{array}{c}
\frac{3}{2}, \frac{3}{2}, 1,1 ; \\
n+\frac{5}{2}, 2,2 ;
\end{array}\right] .
$$

In conclusion of this section, we shall obtain a new formula for $G_{n}$ given by (16) below. It follows from (15), since, by making use of the following well-known identity of the Psi function [14, p. 760]:

$$
\psi(n+z)=\psi(z)+\sum_{m=0}^{n-1} \frac{1}{m+z},
$$

we have

$$
\psi\left(n+\frac{5}{4}\right)-\psi\left(\frac{5}{4}\right)=4 \sum_{m=0}^{n-1} \frac{1}{4 m+5}
$$


and

$$
\psi\left(n+\frac{3}{2}\right)-\psi\left(\frac{3}{2}\right)=2 \sum_{m=0}^{n-1} \frac{1}{2 m+3}
$$

so that

$$
\psi\left(n+\frac{5}{4}\right)=\psi\left(n+\frac{3}{2}\right)+2-\log 2-\frac{\pi}{2}+2 \sum_{m=0}^{n-1} \frac{1}{(2 m+3)(4 m+5)},
$$

since (see $[14$, p. 761$])$

$$
\psi\left(\frac{3}{2}\right)=-\gamma-2 \log 2+2 \quad \text { and } \quad \psi\left(\frac{5}{4}\right)=-\gamma-\frac{\pi}{2}-3 \log 2+4 .
$$

This completes the proof of Proposition 10 below.

Proposition 10. Let $\gamma$ and $\psi(x)$ have the same meaning as above. Then

$$
G_{n}=\frac{1}{\pi} \psi\left(n+\frac{5}{4}\right)+\frac{1}{\pi}(\gamma+4 \log 2)+\Delta_{n},
$$

where

$$
\begin{aligned}
-\pi \Delta_{n}=2 & -\log 2-\frac{\pi}{2}+\sum_{m=0}^{n-1} \frac{2}{(2 m+3)(4 m+5)} \\
& +\frac{1}{4\left(n+\frac{3}{2}\right)}{ }_{4} F_{3}\left[\begin{array}{c}
\frac{3}{2}, \frac{3}{2}, 1,1 ; \\
n+\frac{5}{2}, 2,2 ;
\end{array}\right] .
\end{aligned}
$$

\section{Estimates AND Asymptotics For the LANDAu Constants}

Let

$$
A=\frac{1}{\pi}(\gamma+4 \log 2)=1.06627 \cdots
$$

We now use the closed-form expressions for the Landau constants given by (15) and (16) to establish, in a simple and unified manner, several upper and lower bounds for $G_{n}$ in terms of the Psi and logarithm functions.

Proposition 11. The following two-sided inequality holds true:

$$
A-\delta_{0}+\frac{1}{\pi} \psi\left(n+\frac{3}{2}\right)<G_{n}<A+\frac{1}{\pi} \psi\left(n+\frac{3}{2}\right) \quad\left(n \in \mathbb{N}_{0}\right),
$$


where

$$
\delta_{0}=\frac{2}{\pi}(\log 2+1)-1 \quad \text { and } \quad A-\delta_{0}=1-\frac{1}{\pi}(\gamma+2 \log 2-2) \approx 0.988385 .
$$

Proposition 12. The following two-sided inequality holds true:

$$
A+\frac{1}{\pi} \psi\left(n+\frac{5}{4}\right)<G_{n}<A+\Delta_{0}+\frac{1}{\pi} \psi\left(n+\frac{5}{4}\right) \quad\left(n \in \mathbb{N}_{0}\right)
$$

where

$$
\Delta_{0}=\frac{3}{2}-\frac{1}{\pi}(4+\log 2) \quad \text { and } \quad A+\Delta_{0}=\frac{3}{2}-\frac{1}{\pi}(4-\gamma-3 \log 2) \approx 1.07240 .
$$

Proposition 13. The following inequality holds true:

$$
1+\frac{1}{\pi} \log (n+1) \leqq G_{n}<A+\frac{1}{\pi} \log (n+1) \quad\left(n \in \mathbb{N}_{0}\right)
$$

Proposition 14. The following inequality holds true:

$$
A+\frac{1}{\pi} \log \left(n+\frac{3}{4}\right)<G_{n} \leqq A+\Delta_{0}^{*}+\frac{1}{\pi} \log \left(n+\frac{3}{4}\right) \quad\left(n \in \mathbb{N}_{0}\right),
$$

where

$$
A+\Delta_{0}^{*}=1-\frac{1}{\pi} \log \left(\frac{3}{4}\right) \approx 1.09157 .
$$

Demonstrations. In order to prove Propositions 11 and 12, it is sufficient to show that the sequences $\left\{\delta_{n}\right\}_{n=0}^{\infty}$ and $\left\{\Delta_{n}\right\}_{n=0}^{\infty}$ in (15) and (16) are strictly decreasing. To do so, in the case of $\left\{\Delta_{n}\right\}_{n=0}^{\infty}$ in (16), we need only to verify that

$$
\begin{aligned}
\Delta_{n}-\Delta_{n-1} & =G_{n}-G_{n-1}-\frac{1}{\pi} \frac{4}{4 n+1} \\
& =\left(\frac{(2 n-1) ! !}{(2 n) ! !}\right)^{2}-\frac{1}{\pi} \frac{4}{4 n+1}<0 \quad(n \in \mathbb{N})
\end{aligned}
$$

leads to

$$
\pi<\frac{4}{4 n+1}\left(\frac{(2 n) ! !}{(2 n-1) ! !}\right)^{2} \quad(n \in \mathbb{N}),
$$

and then to appeal to the following Gurland inequalities [9]:

$$
\frac{4 n+3}{(2 n+1)^{2}}\left(\frac{(2 n) ! !}{(2 n-1) ! !}\right)^{2}<\pi<\frac{4}{4 n+1}\left(\frac{(2 n) ! !}{(2 n-1) ! !}\right)^{2} \quad(n \in \mathbb{N}) .
$$


Similarly, we show that the sequence $\left\{\delta_{n}\right\}_{n=0}^{\infty}$ in (15) is strictly decreasing by making use of the well-known Wallis formula:

$$
\frac{2}{2 n+1}\left(\frac{(2 n) ! !}{(2 n-1) ! !}\right)^{2}<\pi<\frac{1}{n}\left(\frac{(2 n) ! !}{(2 n-1) ! !}\right)^{2} \quad(n \in \mathbb{N})
$$

and, thus, demonstrate that the proposed bounds in (18) are valid.

In order to prove Propositions 13 and 14, we consider the following sequences:

$$
\delta_{n}^{*}=\frac{1}{\pi} \log (n+1)+A-G_{n} \quad\left(n \in \mathbb{N}_{0}\right)
$$

and

$$
\Delta_{n}^{*}=G_{n}-\frac{1}{\pi} \log \left(n+\frac{3}{4}\right)-A \quad\left(n \in \mathbb{N}_{0}\right),
$$

which, respectively, follow from (15) and (16) in conjunction with

$$
\psi\left(n+\frac{3}{2}\right) \sim \log (n+1) \quad(n \rightarrow \infty)
$$

and

$$
\psi\left(n+\frac{5}{4}\right) \sim \log \left(n+\frac{3}{4}\right) \quad(n \rightarrow \infty)
$$

which are obtained by means of the following asymptotic formula:

$$
\psi\left(z+\frac{1}{2}\right)=\log z+O\left(\frac{1}{z^{2}}\right) \quad(z \rightarrow \infty)
$$

which, in turn, is readily derivable from the following asymptotic expansion for the psi function [11, p. 33, Eq. (9)]:

$$
\begin{gathered}
\psi(z+a)=\log z-\sum_{m=0}^{n-1}(-1)^{m+1} \frac{B_{m+1}(a)}{m+1} z^{-m-1}+O\left(\frac{1}{z^{n+1}}\right) \quad(z \rightarrow \infty) \\
(|\arg (z)| \leqq \pi-\epsilon \quad(\epsilon>0)),
\end{gathered}
$$

$B_{n}(x)$ being the classical Bernoulli polynomials (see, for example, [17, p. 59 et seq.]).

Now, in order to prove the inequalities (20) and (21) asserted by Propositions 13 and 14, it suffices to show, in a similar fashion as above, that the sequences $\left\{\delta_{n}^{*}\right\}_{n=0}^{\infty}$ and $\left\{\Delta_{n}^{*}\right\}_{n=0}^{\infty}$ in (23) and (24) are strictly decreasing by using the inequalities in (22). 
We note that the estimates in (18) and (19) were found by Cvijovic and Klinowski[4], whileFalaleev [7] and Brutman [3] obtained (20) and (21), respectively.

It can be shown, by a simple numerical computation, that (for all sufficiently large $n$ ) the lower bound in (20) improves the one given in (21), whereas the upper bound given in (21) is better than the one in (20). We thus have arrived at the new estimates contained in Proposition 15 below.

Proposition 15. The following inequality holds true:

$$
1+\frac{1}{\pi} \log (n+1) \leqq G_{n} \leqq 1-\frac{1}{\pi} \log \left(\frac{3}{4}\right)+\Delta_{0}^{*}+\frac{1}{\pi} \log \left(n+\frac{3}{4}\right) \quad\left(n \in \mathbb{N}_{0}\right) .
$$

We now state (without proof) a recent result of Alzer [1, p. 218, Theorem 1]. Indeed, as noted already by Alzer [1], the upper and lower bounds for $G_{n}$ given by (25) improve the bounds presented in (18) to (21).

Proposition 16. Let $\psi^{-1}(z)$ denote the inverse function of $\psi(z)$. Then

$$
A+\frac{1}{\pi} \psi(n+\alpha)<G_{n}<A+\frac{1}{\pi} \psi(n+\beta) \quad\left(n \in \mathbb{N}_{0}\right),
$$

with the following best possible constants:

$$
\alpha=\frac{5}{4} \quad \text { and } \quad \beta=\psi^{-1}(\pi(1-A)) \approx 1.26621 \cdots .
$$

In conclusion, we remark that Landau [10] first studied the asymptotic behavior of $G_{n}$ and showed that

$$
G_{n} \sim \frac{1}{\pi} \log n \quad(n \rightarrow \infty),
$$

while Watson [20, p. 315, Eq. (8)] proved the following asymptotic expansion:

$$
G_{n}=\frac{1}{\pi} \log (n+1)+A-\frac{1}{4 \pi(n+1)}+O\left(\frac{1}{n^{2}}\right) \quad(n \rightarrow \infty) .
$$

In this connection, we find it to be worthwhile to restate the results asserted by Propositions 11 to 14 as follows.

Proposition 17. Assume that $n \in \mathbb{N}_{0}$ and let $A$ be a constant given by (17). Then

$$
\begin{aligned}
& \text { (a) } \quad G_{n} \sim \frac{1}{\pi} \psi\left(n+\frac{3}{2}\right)+A \quad(n \rightarrow \infty) \text {; } \\
& \text { (b) } \quad G_{n} \sim \frac{1}{\pi} \psi\left(n+\frac{5}{4}\right)+A \quad(n \rightarrow \infty) \text {; } \\
& \text { (c) } \quad G_{n} \sim \frac{1}{\pi} \log (n+1)+A \quad(n \rightarrow \infty) \text {; }
\end{aligned}
$$




$$
\text { (d) } \quad G_{n} \sim \frac{1}{\pi} \log \left(n+\frac{3}{2}\right)+A \quad(n \rightarrow \infty) .
$$

Finally, it is not difficult to show that $\delta_{n}$ in (15) is of the following form:

$$
\delta_{n}=\left(\frac{1}{2}\right) \frac{1}{1 \cdot(2 n+3)}+\left(\frac{1 \cdot 3}{2 \cdot 4}\right) \frac{1 \cdot 3}{2 \cdot(2 n+3)(2 n+5)}+\cdots
$$

so that, by making use of (15) and (16), we can deduce the (presumably) new asymptotic expansions asserted by Proposition 18 below.

Proposition 18. Let A be a constant given by (17). Then

$$
\begin{aligned}
& \text { (a) } G_{n}=\frac{1}{\pi} \psi\left(n+\frac{3}{2}\right)+A-\frac{1}{2 \pi(2 n+3)}+O\left(\frac{1}{n^{2}}\right) \quad(n \rightarrow \infty) ; \\
& \text { (b) } G_{n}=\frac{1}{\pi} \psi\left(n+\frac{5}{4}\right)+A-\frac{1}{2 \pi(2 n+3)}-\frac{B}{\pi}+O\left(\frac{1}{n^{2}}\right) \quad(n \rightarrow \infty),
\end{aligned}
$$

where

$$
B=2-\log 2-\frac{\pi}{2}+\sum_{m=0}^{n-1} \frac{2}{(2 m+3)(4 m+5)}
$$

\section{ACKNOWLEDGMENTS}

The present investigation was supported by the Ministry of Science and Environmental Protection of the Republic of Serbia under Research Project Number 144004 and the Natural Sciences and Engineering Research Council of Canada under Grant OGP0007353.

\section{REFERENCES}

1. H. Alzer, Inequalities for the constants of Landau and Lebesgue, J. Comput. Appl. Math., 139 (2002), 215-230.

2. H. Alzer, D. Karayannakis and H. M. Srivastava, Series representations for some mathematical constants, J. Math. Anal. Appl., 320 (2006), 145-162.

3. L. Brutman, A sharp estimate of the Landau constants, J. Approx. Theory, 34 (1982), 217-220.

4. D. Cvijovic and J. Klinowski, Inequalities for the Landau constants, Math. Slovaca, 50 (2000), 159-164.

5. J. Dutka, Two results of Ramanujan SIAM J. Math. Anal. 12 (1981), 471-476. 
6. A. Eisinberg, G. Franze and N. Salerno, Asymptotic expansion and estimate of the Landau constant, Approx. Theory Appl. (New Ser), 17 (2001), 58-64.

7. L. P. Falaleev, Inequalities for the Landau constants, Siberian Math. J., 32 (1991), 896-897.

8. S. Finch, Mathematical Constants, Cambridge University Press, London, 2003.

9. J. Gurland, On Wallis' formula, Amer. Math. Monthly, 63 (1956), 643-645.

10. E. Landau, Abschätzung der Koeffizientensumme einer Potenzreihe Arch. Math. Phys., 21 (1913), 42\{-50, 250\{-255; also in Collected Works, Vols. 5 and 6 (L. Mirsky, I. J. Schoenberg, W. Schwarz and H. Wefelscheid, Editors), Thales Verlag, 1985, pp. 432-440 and 11-16.

11. Y. L. Luke, The Special Functions and Their Approximations, Vol. I, Academic Press, New York and London, 1969.

12. T. M. Mills and S. J. Smith, On the Lebesgue function for Lagrange interpolation with equidistant nodes, J. Austral. Math. Soc. (Ser. A), 52 (1992), 111-118.

13. E. Montaldi and G. Zucchelli, Some formulas of Ramanujan, revisited, SIAM J. Math. Anal., 23 (1992), 562-569.

14. A. P. Prudnikov, Yu. A. Brychkov and O. I. Marichev, Integrals and Series, Vol. 3: More Special Functions, Gordon and Breach Science Publishers, New York, London and Tokyo, 1989.

15. S. Ramanujan, Collected Papers of Srinivasa Ramanujan, (G. H. Hardy, P. V. Seshu Aiyar and B. M. Wilson, Editors), American Mathematical Society and Chelsea Publications, New York, 2000.

16. A. Schonhage, Fehlerfortpflanzung bei Interpolation, Numer. Math., 3 (1961), 62-71.

17. H. M. Srivastava and J. Choi, Series Associated with the Zeta and Related Functions, Kluwer Academic Publishers, Dordrecht, Boston and London, 2001.

18. H. M. Srivastava and H. L. Manocha, A Treatise on Generating Functions, Halsted Press (Ellis Horwood Limited, Chichester), John Wiley and Sons, New York, Chichester, Brisbane and Toronto, 1984.

19. G. N. Watson, Theorems stated by Ramanujan (VIII): Theorems on divergent series, J. London Math. Soc., 4 (1929), 82-86.

20. G. N. Watson, The constants of Landau and Lebesgue, Quart. J. Math. Oxford (Ser. 1), 2 (1930), 310-318.

21. J. E. Wilkins, The Landau constants, in Progress in Approximation Theory (P. Nevai and A. Pinkus, Editors), pp. 829-842, Academic Press, Boston, 1991. 
Djurdje Cvijovic

Atomic Physics Laboratory,

Vinca Institute of Nuclear Sciences,

P. O. Box 522,

YU-11001 Belgrade,

Republic of Serbia

E-mail: djurdje@vin.bg.ac.yu

H. M. Srivastava

Department of Mathematics and Statistics,

University of Victoria,

Victoria, British Columbia V8W 3R4,

Canada

E-mail: harimsri@math.uvic.ca 